\title{
Horner Syndrome Following Attempted Internal Jugular Catheter Placement in a Toddler With Congenital Heart Disease
}

\author{
Joseph D. Tobias ${ }^{\mathrm{a}, \mathrm{b}, \mathrm{c}, \mathrm{e}}$, Brian F. Joy ${ }^{\mathrm{c}, \mathrm{d}}$
}

\begin{abstract}
Horner syndrome results from interruption of the normal sympathetic innervation to the face and eye. Common clinical manifestations include ptosis, miosis, anhidrosis, conjunctival injection and relative enophthalmos. Several diverse etiologies may be responsible for Horner syndrome. We present an 8-month-old child who was noted to have Horner syndrome following attempts at placement of a right jugular venous catheter. The normal sympathetic innervation of the head and eye are presented, potential etiologies for Horner syndrome are reviewed and previous reports of Horner syndrome following invasive procedures are presented.
\end{abstract}

Keywords: Horner syndrome; Internal jugular catheter placement; Toddler; Congenital heart disease

\section{Introduction}

Horner syndrome (also known as Bernard-Horner syndrome or oculosympathetic palsy) is a clinical disorder resulting from interruption of the normal sympathetic innervation to the face and eye [1]. The clinical presentation includes ptosis, miosis, anhidrosis, conjunctival injection and rela-

Manuscript accepted for publication May 6, 2014

${ }^{a}$ Department of Anesthesiology \& Pain Medicine, Nationwide Children's Hospital, Columbus, Ohio, USA

${ }^{\mathrm{b}}$ Department of Anesthesiology \& Pain Medicine, The Ohio State

University College of Medicine, Columbus, Ohio, USA

${ }^{c}$ Department of Pediatrics, The Ohio State University College of

Medicine, Columbus, Ohio, USA

${ }^{\mathrm{d}}$ Division of Pediatric Cardiology, Nationwide Children's Hospital, Columbus, Ohio, USA

${ }^{\mathrm{e}}$ Corresponding Author: Joseph D. Tobias, Department of

Anesthesiology \& Pain Medicine, Nationwide Children's Hospital,

700 Children's Drive, Columbus, Ohio 43205, USA.

Email: Joseph.Tobias@Nationwidechildrens.org

doi: http://dx.doi.org/10.14740/jmc1795w tive enophthalmos. The disorder results from interruption of the normal sympathetic pathway at the first-, second- or third-order neuron [2]. Central lesions may involve the firstorder neuron; pre-ganglionic lesions such as a lung tumor can compress the second-order neuron, while involvement of the third-order neuron may occur in the area near the stellate ganglion and around the great vessels of the neck. When there is a question regarding the diagnosis and the location of the lesion causing Horner syndrome, radiographic imaging or the response to ophthalmic medications (cocaine and $\alpha$-adrenergic agonists) may be used to localize the lesion [3]. Various infectious, neoplastic and vascular pathologies may result in Horner syndrome, the spectrum ranging from lifethreatening to benign.

Given its location deep to the vascular structures of the neck, the stellate ganglion and the sympathetic chain may be damaged during attempts at cannulation of the internal jugular vein $[4,5]$. We present an 8-month-old child who was noted to have Horner syndrome following attempts at placement of a right jugular venous catheter. The normal sympathetic innervation of the head and eye is presented, potential etiologies for Horner syndrome are reviewed and previous reports of Horner syndrome following invasive procedures are presented.

\section{Case Report}

Institutional Review Board approval for isolated case reports is not required at Nationwide Children's Hospital, Columbus, Ohio. The patient was an 8-month-old, $5.4 \mathrm{~kg}$ toddler with a history of an atrial septal defect, pulmonary stenosis and a small patent ductus arteriosus. She was admitted to an outside hospital for treatment of bronchiolitis and respiratory failure related to respiratory syncytial virus. She required endotracheal intubation and mechanical ventilation for 5 - 6 days. Following tracheal extubation, she was noted to anisocoria with the right pupil being $4-5 \mathrm{~mm}$ while the left was $2-3 \mathrm{~mm}$. Both were reactive to light with no other focal neurologic findings. Due to parental concerns, she was transferred to our institution for further investigation and treatment. On arrival, she was on continuous positive airway 
pressure (CPAP) which was transitioned to high flow nasal cannula for the treatment of her bronchiolitis. Physical exam revealed an alert infant with no focal neurologic findings. The left pupil was $2-3 \mathrm{~mm}$ while the right was $4-5 \mathrm{~mm}$. Both were reactive to light. She was also noted to have slight ptosis of the right eyelid. Based on her physical findings, a diagnosis of Horner syndrome was made. Computed tomography scan of the head was normal. Additional findings on physical examination included 6 - 8 old puncture marks over the right side of the neck. On review of the medical records from the outside hospital, there had been a prolonged and subsequently aborted attempt at placement of a central venous catheter into the right internal jugular vein. There was no mention of the use of ultrasound during the procedure in the medical records. Further imaging at our institution included an ultrasound of the right neck which revealed no evidence of hematoma and normal architecture of the carotid artery and internal jugular vein. Three weeks after the event, there has been slight resolution of the ptosis, but continued anisocoria.

\section{Discussion}

Unlike the parasympathetic innervation of the head and neck, the sympathetic nerves exit the central nervous system from the thoracic spinal cord. They must therefore travel cephalad back into the head and neck region to exert their influence. The axon of the first-order neuron of the sympathetic fibers, the cell bodies of which are located in the midbrain (hypothalamus), travel caudad through the brain stem and into the spinal cord to the thoracic level. These fibers do not cross the midline, synapsing in the intermediolateral gray column of the thoracic spinal cord at the level of C7-T3 with the cell body of the second-order neuron. The second-order neuron (pre-ganglionic fibers) exits the thoracic spinal cord travelling in white rami communicantes, passes over the apex of lung within the thoracic cavity and ascends into the neck through the thoracic sympathetic ganglia (stellate ganglion), synapsing with the cell body of the third-order neuron in the superior cervical ganglion. From there, the third-order neuron (post-ganglionic fibers) travels alongside the great vessels in the neck, enters the cranium, and then the orbit to innervate the eye. Sympathetic innervation of the eye includes the dilator pupillae muscle and the levator palpebrae superioris (Muller's muscle). Damage at any point along this pathway can result in Horner syndrome.

Various etiologies for Horner syndrome exist including infectious, vascular, neoplastic and traumatic causes. Given the pathway of the sympathetic nervous system, these lesions may be found within the brain, the spinal cord or the peripheral sympathetic pathway. Regardless of the site of the pathology, the clinical symptoms remain constant. Although uncommon, there are numerous reports in the literature dat- ing back into the 1970s regarding Horner syndrome following attempts at placement of an internal jugular catheter [6, 7]. Other procedural-related causes of Horner syndrome have included tube thoracostomy placement, subclavian venous catheterization and epidural anesthesia as well as various surgical procedures of the neck [8-11].

A literature search revealed that there are 15 previously reported cases of Horner syndrome attributed to cannulation of the internal jugular vein in pediatric patients [6, 7, 12-23]. The age range was 8 months to 17 years. These are mainly from case reports, but the development of Horner syndrome has also been described in two retrospective analyses [12, 13] and two prospective studies [7, 14] focusing on internal jugular cannulation in pediatric patients. Eleven of the reports describe difficulty accessing the internal jugular vein with multiple attempts required [13-22]. Three cases of Horner syndrome occurred despite the use of ultrasound guidance for cannulation $[12,17,18]$. Although two of these ultrasound-guided cases were described as difficult access $[17,18]$, five cases were associated with hematoma formation at the site of cannulation $[13,14,16,17,21]$. Nine of the patients were described as having complete recovery of the symptoms of Horner syndrome in from $24 \mathrm{~h}$ to 5 months [7, $12,15-17,19,20,22]$.

Treatment involves general supportive care; however, imaging may be indicated if there is a question regarding the etiology. In our patient, given the concerns regarding the larger left pupil, computed tomography of the brain was obtained to rule out intracranial pathology [24]. However, on further examination, it was determined that the pupillary abnormality was in fact a miotic right pupil. As was done in our patient, ultrasound imaging of the vasculature of the neck seems indicated as pseudoaneurysms have been reported as a complication of internal jugular line placement [25]. Most importantly, prevention remains the primary intervention in such cases. The sympathetic chain and the vascular structures are in close proximity within the neck. The cell bodies of post ganglionic sympathetic fibers are located within one of three ganglia within the neck (superior, middle and inferior cervical ganglion). In the majority of cases, the inferior cervical ganglion fuses with the first thoracic ganglia forming the stellate ganglion which lies anterior to the transverse process of the seventh cervical vertebra and just deep to the carotid artery and the internal jugular vein. Furthermore, the literature demonstrates variability in the anatomy and position of the great vessels in the neck. In a prospective investigation of two-dimensional suing ultrasound of the internal jugular, subclavian and femoral vein of 142 infants and children, there was a $7.7 \%$ variation for the internal jugular with the most common being a lateral or anterior position of the internal jugular vein in relationship to the carotid artery [26]. Ultrasound has also been shown to facilitate central venous catheter placement with fewer attempts, shorter time to catheter placement, fewer carotid punctures and a higher success 
rate $[27,28]$.

In summary, we present the development of Horner syndrome following attempted cannulation of the jugular vein in an infant. An appropriate diagnosis may limit the need for unnecessary imaging; however, if the etiology is in doubt, imaging is suggested to identify pathologic lesions along the sympathetic pathway. Given the proximity of structures within the neck especially in a child, we would highly recommend the use of ultrasound to facilitate central venous catheter placement. Ultrasound to facilitate central venous access was first reported in 1986. The literature clearly demonstrates that ultrasound-guided vascular access is being widely adopted as a technique that facilitates successful completion of the procedure while limiting the potential for adverse effects and is recognized by many as the standard of care for such procedures.

\section{References}

1. Walton KA, Buono LM. Horner syndrome. Curr Opin Ophthalmol. 2003;14(6):357-363.

2. Lee JH, Lee HK, Lee DH, Choi CG, Kim SJ, Suh DC. Neuroimaging strategies for three types of Horner syndrome with emphasis on anatomic location. AJR Am J Roentgenol. 2007;188(1):W74-81.

3. Mughal M, Longmuir R. Current pharmacologic testing for Horner syndrome. Curr Neurol Neurosci Rep. 2009;9(5):384-389.

4. Lazar I, Cavari Y, Rosenberg E, Knyazer B. Horner's syndrome in patients admitted to the paediatric intensive care unit: epidemiology, diagnosis and clinical practice. Anaesth Intensive Care. 2013;41(1):20-23.

5. Kong YX, Wright G, Pesudovs K, O'Day J, Wainer Z, Weisinger HS. Horner syndrome. Clin Exp Optom. 2007;90(5):336-344.

6. Parikh RK. Horner's syndrome. A complication of percutaneous catheterisation of internal jugular vein. Anaesthesia. 1972;27(3):327-329.

7. Prince SR, Sullivan RL, Hackel A. Percutaneous catheterization of the internal jugular vein in infants and children. Anesthesiology. 1976;44(2):170-174.

8. Thomas DT, Dagli TE, Kiyan G. Horner's syndrome as a rare complication of tube thoracostomy: case reports and review of literature. J Pediatr Surg. 2013;48(6):14291433.

9. Sulemanji DS, Candan S, Torgay A, Donmez A. Horner syndrome after subclavian venous catheterization. Anesth Analg. 2006;103(2):509-510.

10. Barbara R, Tome R, Barua A, Barbara A, Gaitini L, Odeh M, Garzozi H. Transient Horner syndrome following epidural anesthesia for labor: case report and review of the literature. Obstet Gynecol Surv. 2011;66(2):114119.
11. Allen AY, Meyer DR. Neck procedures resulting in Horner syndrome. Ophthal Plast Reconstr Surg. 2009;25(1):16-18.

12. Liberman L, Hordof AJ, Hsu DT, Pass RH. Ultrasoundassisted cannulation of the right internal jugular vein during electrophysiologic studies in children. J Interv Card Electrophysiol. 2001;5(2):177-179.

13. Guccione P, Gagliardi MG, Bevilacqua M, Parisi F, Marino B. Cardiac catheterization through the internal jugular vein in pediatric patients. An alternative to the usual femoral vein access. Chest. 1992;101(6):1512-1514.

14. Garcia EG, Wijdicks EF, Younge BR. Neurologic complications associated with internal jugular vein cannulation in critically ill patients: a prospective study. Neurology. 1994;44(5):951-952.

15. Suominen PK, Korhonen AM, Vaida SJ, Hiller AS. Horner's syndrome secondary to internal jugular venous cannulation. J Clin Anesth. 2008;20(4):304-306.

16. Ahmad M, Hayat A. Horner's syndrome following internal jugular vein dialysis catheter insertion. Saudi J Kidney Dis Transpl. 2008;19(1):94-96.

17. Silvani P, Valeri R, Corbella E, Torri G. [Claude Bernard-Horner syndrome following percutaneous catheterization of the jugular vein in an infant]. Cah Anesthesiol. 1993;41(3):266-268.

18. Ford S, Lauder G. Case report of Horner's syndrome complicating internal jugular venous cannulation in a child. Paediatr Anaesth. 2007;17(4):396-398.

19. Williams MA, McAvoy C, Sharkey JA. Horner's syndrome following attempted internal jugular venous cannulation. Eye (Lond). 2004;18(1):104-106.

20. Peake ST, Bollen B. Unilateral fixed dilated pupil after aortic valve replacement: an unusual combination of causes. J Cardiothorac Anesth. 1990;4(6):737-739.

21. Fita G, Basora M, Jimenez MJ, Mulet J. [Horner's syndrome: an infrequent complication of cannulation of the internal jugular vein]. Rev Esp Anestesiol Reanim. 1990;37(2):111-112.

22. Mostaza AG, Molina E, Cebrian J. [Horner syndrome prolonged after canalization of internal jugular vein]. Rev Esp Anestesiol Reanim. 1996;43(4):151-152.

23. Buyuktortop N, Gurbuz-Koz O, Atalay T, Kural G. A case of postganglionic Horner syndrome after catheterization of internal jugular vein confirmed with pharmacological tests. Turk J Pediatr. 2008;50(4):391-394.

24. Al-Moosa A, Eggenberger E. Neuroimaging yield in isolated Horner syndrome. Curr Opin Ophthalmol. 2011;22(6):468-471.

25. Mastan M. Carotid artery pseudoaneurysm with Horner's syndrome: delayed complication of internal jugular venous cannulation. Hosp Med. 2005;66(5):314-315.

26. E PSN, Grousson S, Duflo F, Tahon F, Mottolese C, Dailler F. Ultrasonographic anatomic variations of the major veins in paediatric patients. Br J Anaesth. 
2014;112(5):879-884.

27. Verghese ST, McGill WA, Patel RI, Sell JE, Midgley FM, Ruttimann UE. Ultrasound-guided internal jugular venous cannulation in infants: a prospective comparison with the traditional palpation method. Anesthesiology.
1999;91(1):71-77.

28. Verghese ST, McGill WA, Patel RI, Sell JE, Midgley FM, Ruttimann UE. Comparison of three techniques for internal jugular vein cannulation in infants. Paediatr Anaesth. 2000;10(5):505-511. 